\title{
Nonlinear Bio-photonic Crystal Effect of Silica Deposition in Plants -
}

\author{
P. C. Cheng ${ }^{1 *}$, C. K. Sun ${ }^{2}$, W. Y. Cheng ${ }^{3}$ and D. B. Walden ${ }^{4}$ \\ ${ }^{1}$ Division of Bio-engineering, Department of Electrical and Computer Engineering and \\ Department of Radiology, National University of Singapore, Singapore \\ ${ }^{2}$ Dept. of Electrical Engineering, National Taiwan University, Taipei, Taiwan, Rep. of China \\ ${ }^{3}$ Dept. of Genetics, University of Wisconsin, Madison, WI 53703 USA \\ ${ }^{4}$ Dept. of Plant Sciences, University of Western Ontario, London, Ontario, Canada
}

Silica deposits are commonly found in crop plants such as rice, wheat and maize. This amorphous silica can be found in most of the epidermal cell wall and in specialized dumbbell-shaped epidermal cells (Si). The silica deposits found in the dumbbell-shaped cell is frequently referred as biological opals. These deposits have a refractive index of around 1.4. The silica contents can reach as high as $30 \%$ in dry ash weight in certain rice variety and is believed to be responsible for the rigidity of the plant and resistance to pathogens. Due to hard and brittle nature of the silica deposit as well as its high refractive index $(\eta=1.42-1.44$ [1]), the technical difficulties encountered in the study of silica cell are similar to the study of hard tissues (tooth and bone) in animal system. The silica depositions in plant tissue also pose a significant problem in ultra-sectioning for electron microscopy. Using confocal microscopy and X-ray microradiography, we have been able to gain insight in the structure of silica cells. In maize, silica cells are generally found on the adaxial surface of the leaf (Figure 1,2,4), abaxial surface of leaf sheath (Figure 3), the surface of the stem (excluding the nodal region) and in adaxial surface of glume and husk. X-ray contact microradiography shows X-ray dense deposition in epidermal cell wall (Figure 8) and in specific dumbbell shaped silica cells located above minor veins (Figure 2, 3, 7; V). SEM image (Figure 3, LLE mode) and replica of leaf sheath surface (Figure 2) reveal the location and distribution of the silica cells. EDS image correlates well with the X-ray microradiograph indicating that the X-ray dense material are indeed containing Si. In confocal microscopy, the outline of the silica cell is revealed as the silica deposits show no autofluorescent (Figure 1, 4); since the specimen was mounted in FocusClear $(\eta=1.40$, Pacgen Biopharmaceuticals, Vancouver, Canada) which nearly matches the refractive index of the silica deposits, very little back-scattered signal was detected. However, in a two-channel confocal image of a methyl salicylate $(\eta=1.535-1.538)$ mounted specimen, the silica deposition can be seen in back scattering mode (red), the cell nucleus (N) was stained with Feulgen and imaged in fluorescent mode (blue). Silica cell can be isolated together with the epidermal cuticle by the treatment of $\mathrm{ZnCl}_{2}-\mathrm{HCl}$ mixture [2, 3]. After chemical isolation, the silica deposits are attached to the continuous cuticular sheet. The dumbbell shaped silica cell exhibits strong birefringence properties and the retardeance and slow-axis can be determined by the use of dynamic polarization microscope (Pol-Scope, CRI, Cambridge, MA). It was reported that the dumbbell-shaped silica cell of rice (Oryza sativa) exhibits strong polarization-dependent SHG properties (Figure 6). It is believed to be the result of concentrically orientated silica deposition in the opaline silica. The term of biological photonic crystal-like structure was use to described the strong SHG properties of the opaline silica. Maize silica cell also exhibits similar nonlinear properties.

[1] Jones and Handreck, Adv. Agron, 19 (1967), 107-149.

[2] P. C Cheng et al., Can. J. Bot. (1986)64: 2088-2079.

[3] P. C Cheng et al., Natl. Sci. Counc. Month, ROC, 9 (1981) 983-994.

[4] S. W. Chu et al., J. Microscopy, 208 (2002), 190-200.

*On leave from the Department of Electrical Eng., State Univ. of New York at Buffalo, USA 

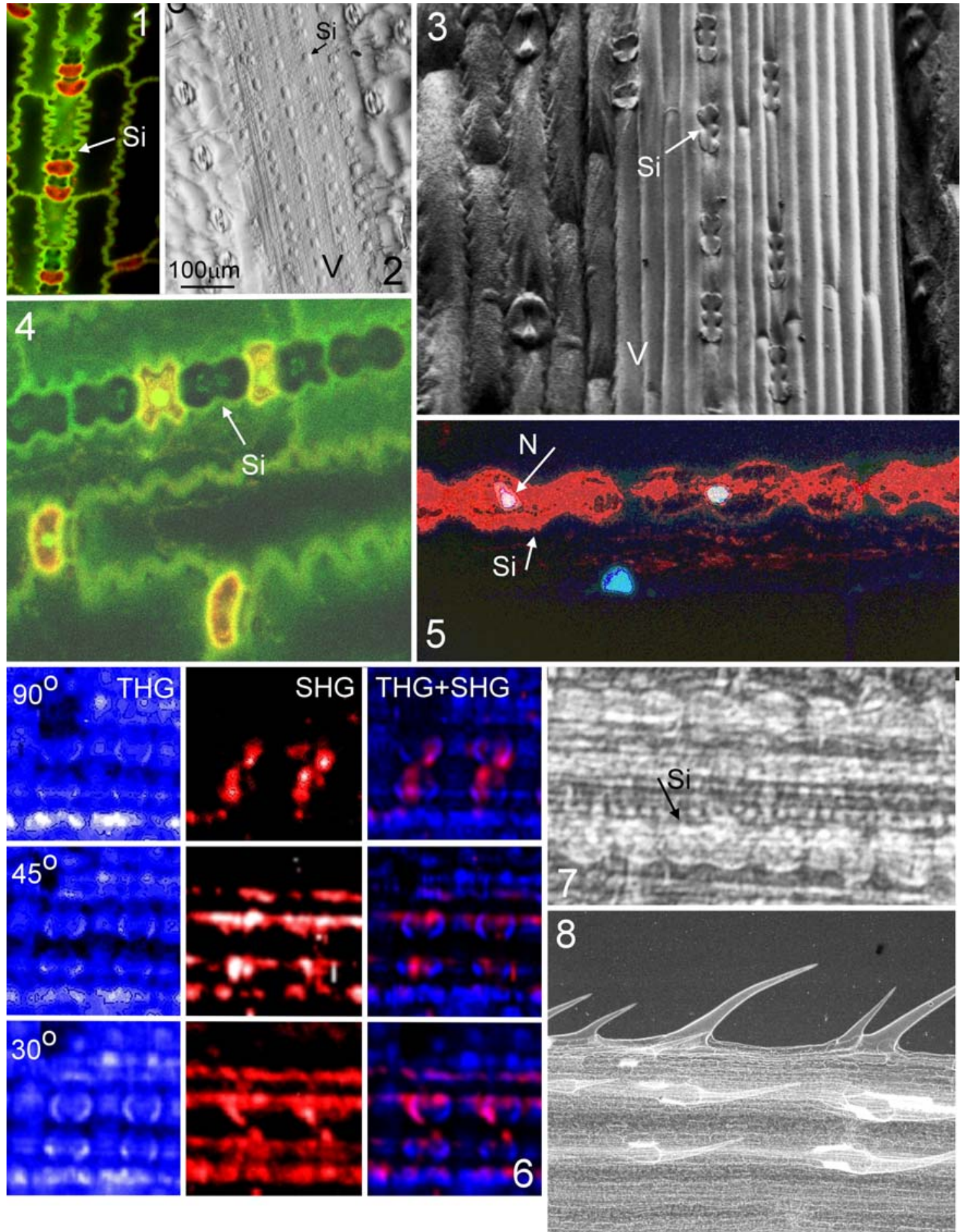

FIG 1. Confocal image of maize epidermal cells showing silica cell (Si)

FIG 2. Surface replica of maize leaf sheath.

FIG 3. SEM image of abaxial surface of leaf sheath (LLE mode).

FIG 4. Confocal image of maize leaf silica cells (red: auto-fluorescent, green: back scatter light) FIG 5. Confocal image showing silica cells. Green: auto-fluorescent, red: back scatter light) FIG 6. Polarization dependent SHG signal from rice leaf. THG: third harmonic generation FIG 7, 8. X-ray microradiograph of maize leaf showing silica deposition (pulse X-ray imaging) 
https://doi.org/10.1017/S1431927603446771 Published online by Cambridge University Press 